\title{
Coupled surface plasmon polaritons on thin metal slabs corrugated on both surfaces
}

\author{
I. R. Hooper and J. R. Sambles \\ Thin Film Photonics, School of Physics, University of Exeter, Stocker Road, Exeter, Devon, EX4 4QL, United Kingdom
}

(Received 11 December 2003; published 28 July 2004)

\begin{abstract}
A modelling study of the effect of coupled surface plasmon polaritons (SPPs) on the optical response of a thin metal film corrugated on both surfaces is presented. Initially the case of conformally corrugated metal films (the corrugations on each surface are identical and in phase with each other) is considered. Sinusoidal structures, and those having an additional first harmonic, $2 k_{g}$ (where $k_{g}$ is the grating vector) component, are investigated. This $2 k_{g}$ component opens up significant band gaps in the SPP dispersion curves, and also causes anticrossing behavior between the long range SPPs and short range SPPs. It is shown that this anticrossing, and the band gap, have a common explanation. Following this, nonconformally corrugated films are examined, and strongly enhanced resonant transmission is shown to occur, which can be almost independent of the in-plane wave vector. The results presented show that, though the enhanced transmission through hole arrays which has provoked extensive recent investigation is of great interest from a physics viewpoint, other structures which exhibit enhanced transmission may provide more benefits, including higher transmission, for some applications.
\end{abstract}

DOI: 10.1103/PhysRevB.70.045421 PACS number(s): 73.20.Mf, 42.70.Qs, 78.66.Bz, 41.20.Jb

\section{INTRODUCTION}

Interest in enhanced transmission of radiation through metal films has increased in recent years due to the discovery of unexpectedly high transmission of light through optically thick metal films perforated with arrays of holes. ${ }^{1,2}$ This enhanced transmission through hole arrays has been attributed to the excitation of surface plasmon polaritons (SPPs) on either side of the metal film coupling together through the holes. ${ }^{3,4}$ Work has also been performed on thick metal films perforated with periodic arrays of slits, where unusually high transmission also occurs. ${ }^{5-7}$

Enhanced transmission has also been observed through corrugated optically thin metal films ( $<100 \mathrm{~nm}$ thick). This can occur whether the dielectrics bounding the metal film are dissimilar, in which case the two different SPPs on either surface may separately increase the transmission under certain circumstances, ${ }^{8,9}$ or if they are identical, in which case coupled SPPs may enhance the transmission. ${ }^{10}$ However, no extensive study into this enhanced transmission via the excitation of coupled SPPs has been performed, and it is this that is the focus of the present paper. The case of coupled SPPs excited on thin metal films which are corrugated on both sides is considered here, and the optical response of these structures are obtained through theoretical modelling. Particular emphasis is paid to the effect of the phase between the two corrugations, since some similarities with the results obtained for the transmission through metal slits may be evident (in the case of the two corrugations being in antiphase).

A SPP is a longitudinal surface charge density oscillation coupled to EM fields, which may be excited at the interface between a metal and a dielectric. ${ }^{11}$ The fields of the SPP decay exponentially into both bounding media, although due to the high negative permittivity of the metal they decay far more rapidly into the metal than into the dielectric. A SPP may not be optically excited at a planar metal surface by incident plane waves without some method of increasing the wave vector of the incident light since the wave vector of the
SPP is beyond the maximum wave vector available to incident light. Also, a SPP can only be excited with TM polarized light, since an orthogonal component of the $E$-field of the incident light at the surface is required to excite the surface charge density oscillation. In this work the case of periodically corrugated metal films are explored, since, above a cut-off wavelength this corrugation allows the SPP to be directly radiatively excited. The TM polarized light is incident in the classical mount where the plane of incidence contains the grating vector (is perpendicular to the grating grooves).

If a thin metal film $(<100 \mathrm{~nm})$ is bounded by dielectrics with identical dielectric functions the SPPs on the two interfaces will be excited at the same frequency (for a given wave vector), and in this case the SPPs on the two interfaces couple together to form two coupled SPPs described as the long range SPP (LRSPP) and the short range SPP (SRSPP). ${ }^{12-16}$ The difference in nature of these two coupled SPP modes arises from the fact that they have different surface charge density distributions. The LRSPP has a charge distribution which is antisymmetric between the top and bottom surfaces (in other words if there is a positive charge density on the top surface there is a corresponding negative charge density on the bottom surface). Conversely, the SRSPP has a charge distribution which is symmetric between the top and bottom surfaces (a positive charge density on the top surface has a corresponding positive charge density on the bottom surface). Due to these different surface charge density distributions, and associated electromagnetic fields, the two modes have different energies, and are therefore excited at different frequencies of incident light (for a given in-plane wave vector).

If the film is corrugated then not only does radiative coupling to the mode become allowed but the added complexities of coupling simultaneously to both surfaces, as well as the potential for grating-induced band-splitting, arises.

The work contained in this paper is split into two main sections. In the first section a conformally corrugated metal 
film is examined in which the corrugations on the two interfaces are identical and in phase with each other, and in the second section nonconformally corrugated metal films are explored. Here the profiles of the two corrugations are identical, but the corrugation on the lower (transmitting) interface is phase shifted with respect to the corrugation on the top interface. In both cases a sinusoidal corrugation, with grating vector $k_{g}$, is investigated before the effect of an additional first harmonic, $2 k_{g}$, component in the grating profile is considered. Thus, the profile describing the structures is given by

$$
\begin{gathered}
\zeta_{1}(x)=a_{1} \sin \left(k_{g} x+\phi\right)+a_{2} \sin \left(2 k_{g} x+\phi \pm \pi / 2\right)+d, \\
\zeta_{2}(x)=a_{1} \sin \left(k_{g} x\right)+a_{2} \sin \left(2 k_{g} x \pm \pi / 2\right),
\end{gathered}
$$

where $\zeta_{1}(x)$ and $\zeta_{2}(x)$ are the profiles of the top (incident) and bottom (transmitting) surfaces, respectively, with the two surfaces being separated by an average thickness $d$. For each of the structures investigated the values of $d, a_{1}, a_{2}$ and the sign of the $\pi / 2$ phase shift of the $2 k_{g}$ component are described where necessary. When no $a_{2}$ value is given for a structure the profile is purely sinusoidal.

\section{COMPUTATIONAL METHOD}

The method used in the computer code for the modelling in this paper is based upon that originally proposed by Chandezon et al. ${ }^{17}$ in which a nonorthogonal curvilinear coordinate transformation is used to map the grating profile onto a flat plane. This enables easier matching of the tangential components of the fields at the boundaries, from which a scattering matrix for the system is obtained. From this, the fields in the two media are determined, and the reflection and transmission coefficients for the various orders calculated. The absorption in the metal can then be calculated as the total incident intensity minus the sum of the intensities of all the propagating diffracted orders. It is also possible to compute the dispersion of any surface modes by identifying peaks in the scattering matrix as a function of frequency and in-plane wave vector. This enables the dispersion of the modes to be obtained in regions of $\omega-k_{x}$ space which would not be possible by investigating the reflectivity from a surface due to the fact that it is not dependent upon the coupling of the surface mode to incident radiation. This method has been reported in more detail elsewhere, ${ }^{18}$ as has the extension which allows the investigation of multilayer and multishape structures. ${ }^{19}$

In the following work the permittivity of the metal gratings is modelled as that of silver, being described by polynomials fitted to experimentally derived values ${ }^{20}$ for both the real and imaginary parts of the permittivity,

$$
\begin{aligned}
\varepsilon_{r}= & -255.3185+1.9863 \times 10^{-13} \omega-6.0794 \times 10^{-29} \omega^{2} \\
& +8.3810 \times 10^{-45} \omega^{3}-4.3004 \times 10^{-61} \omega^{4}, \\
\varepsilon_{i}= & 83.2575-1.3279 \times 10^{-13} \omega+9.0474 \times 10^{-29} \omega^{2} \\
& -3.2880 \times 10^{-44} \omega^{3}+6.6591 \times 10^{-60} \omega^{4}-7.0893 \\
& \times 10^{-76} \omega^{5}+3.0913 \times 10^{-92} \omega^{6}
\end{aligned}
$$

with the bounding dielectrics described as $n=1.0$ for all the modelling in this paper.

\section{COUPLED SPPS ON CONFORMALLY MODULATED THIN METAL SLABS}

\section{A. Sinusoidal corrugations}

The dispersion relation of coupled SPPs on a planar metal slab shows a splitting of the single planar-interface SPP dispersion curve into two separate dispersion curves, which take a similar form to the single metal/dielectric interface case, but with the LRSPP curve shifted up in frequency (for a given in-plane wave vector), and the SRSPP curve shifted down in frequency (for a given in-plane wave vector). When a corrugation is added to the surfaces these two curves are folded back into the region of $\omega-k$ space available to incident radiation (diffractive coupling), and these coupled SPPs may then be excited by incident light.

The size of the splitting between the LRSPP and the SRSPP is predominantly determined by the thickness of the metal slab. This is shown in Fig. 1, in which the zeroth order reflection, transmission (log scale), and absorption of the system are given as a function of frequency and slab thickness for light normally incident $\left(k_{x}=0\right)$ upon a thin silver slab, with both surfaces corrugated identically with a $10 \mathrm{~nm}$ amplitude, $400 \mathrm{~nm}$ pitch, sinusoid.

At large slab thickness $(>100 \mathrm{~nm})$ the two branches of the coupled SPPs converge to the frequency at which the first order SPP would occur on a single interface silver grating having the same parameters. (For a $400 \mathrm{~nm}$ pitch grating it would be expected that the first order SPP would occur, for normal incidence, at approximately $420 \mathrm{~nm}$, or $f$ $\approx 7.1 \times 10^{14} \mathrm{~Hz}$ ). As the slab thickness is reduced it is clear that the LRSPP approaches the diffracted order light line (at $f=7.5 \times 10^{14} \mathrm{~Hz}$ ) asymptotically, and that the SRSPP rapidly reduces in frequency.

In reflection both the SRSPP and the LRSPP are shown as reflectivity minima. This is not surprising since the predominant mechanism leading to this feature is reradiation of the SPPs from the corrugation on the top surface (scattering from the bottom surface will be limited due to attenuation through the silver slab). The field distributions at the top surface for the SRSPP and LRSPP are very similar, and they both result in reflectivity minima since the reradiated light is in antiphase with the specularly reflected light (in the same way as is well known for a single interface metal/dielectric grating ${ }^{11}$ ).

In transmission, the resultant zeroth order transmitted fields will comprise a combination of directly transmitted zeroth order light with diffracted reradiation from the charges on the lower surface. Now, because of the antisymmetric charge distribution for the LRSPP, and the symmetric charge distribution of the SRSPP, the surface fields for the two modes at the lower surface have a relative phase difference of $180^{\circ}$. Consequently reradiation from the lower surface has a $180^{\circ}$ phase difference for the two modes. Because of this, excitation of the two modes will have a different effect on the zeroth order transmitted intensities. This can be seen from Fig. 1(b), where the feature due to the SRSPP 

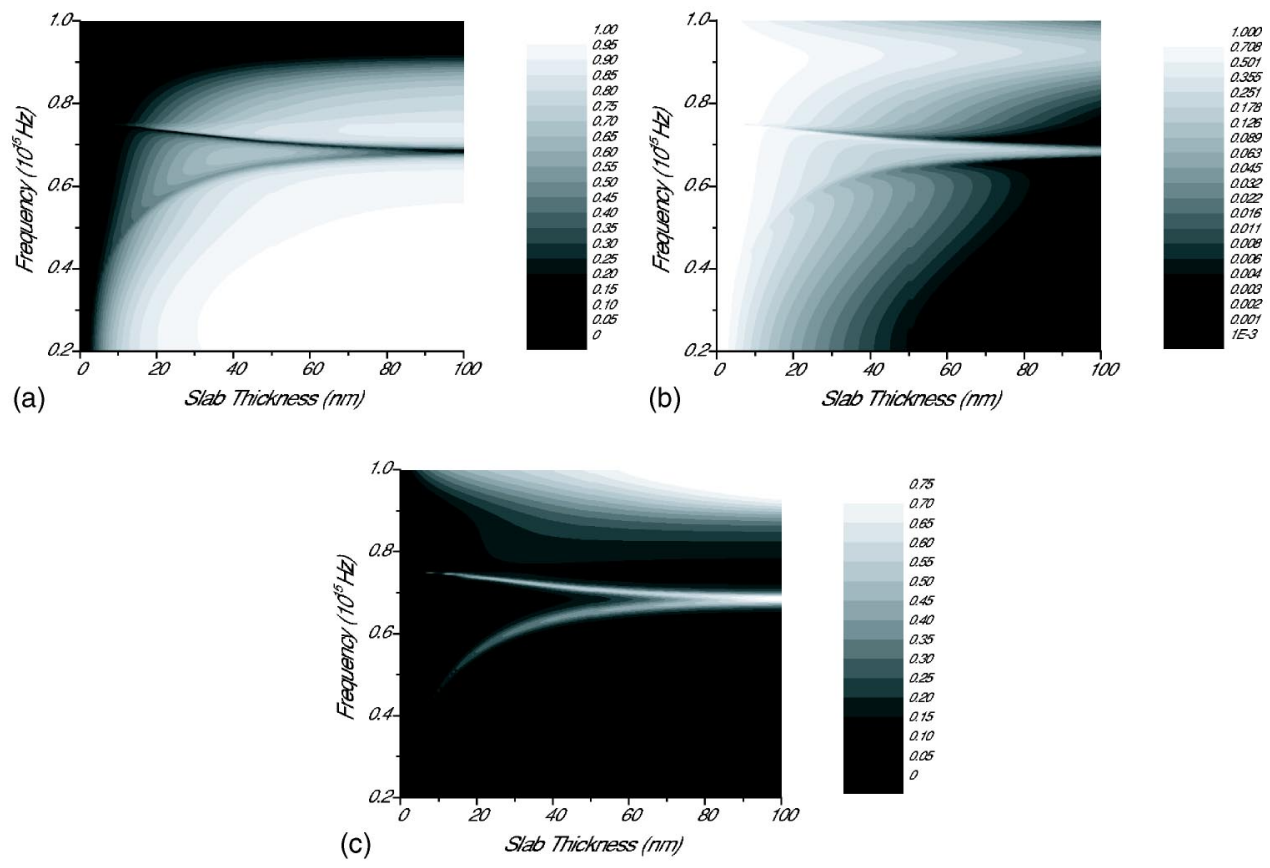

FIG. 1. The optical response as a function of frequency and slab thickness for $k_{x}=0$ on a conformal, sinusoidally corrugated, silver slab of $10 \mathrm{~nm}$ amplitude and $400 \mathrm{~nm}$ pitch, in the classical mount. (a) Reflectivity, (b) transmissivity (log scale), and (c) absorption. is evident as a transmission minimum followed by a maximum (with increasing frequency), whereas the LRSPP shows a transmission maximum followed by a minimum.

In Fig. 2 the zeroth order reflection, transmission, and absorption of the system together with the mode dispersion (obtained from the scattering matrices) are shown as a function of frequency and in-plane wave vector. The slab thickness is fixed at $30 \mathrm{~nm}$, with the rest of the parameters describing the system being the same as for Fig. 1 .

This figure confirms the previous comments regarding the form of the dispersion curve of the long range and short range SPPs on a conformally corrugated thin metal slab. It also shows that the form of the features (whether they are reflectivity or transmissivity maxima or minima) is the same for all values of the in-plane wave vector.

\section{B. Sinusoidal corrugations with an additional $2 k_{g}$ component}

If a first harmonic is added to the grating profile the situation becomes more complicated. When higher harmonics are added to the grating profile on a single interface dielectric/metal grating, band gaps open in the SPP dispersion curves at the points at which the different branches of the curves cross. ${ }^{21}$ The size of the band gaps are predominantly determined by the amplitude of the higher harmonics since it is the first order scattering of the SPP from these which is mainly responsible for their existence, although second or even third order scattering from the fundamental may be significant for very deep gratings. For normally incident light there are then two energies for the SPP, and these correspond to the two possible standing wave SPP solutions with symmetric or antisymmetric surface charge density distributions on either side of the grating peaks. For the lowest energy crossing point at normal incidence it is the $2 k_{g}$ component in the description of the grating profile which predominantly gives rise to the band gap (though a two scatter process from the fundamental $k_{g}$ component also has a small effect), and it is this crossing point which will be considered in this paper. For the other crossing points it is higher harmonics (or multiple scattering from lower harmonics) which give rise to the band gaps.

It is important to note at this point that there are two different symmetries of the surface charge densities discussed in this paper. Previously, when the differences between the surface charge density distributions were discussed for the LRSPP and SRSPP, the symmetry differences between the two modes corresponded to the positions of their surface charge density maxima on either side of the thin metal slab. In the case of discussing the energies of the band edges on either side of a band gap the symmetry refers to the position of their surface charge density maxima on either side of the grating peaks. These two solutions in the latter case (for normally incident light) correspond to the surface charge density maxima of the standing wave states occurring at either the midpoints between the maxima and minima of the overall grating profile (the antisymmetric case), or at the peaks and troughs of the overall grating profile (the symmetric case).

The different energies at the band edges are due to the different positions of the surface charge density maxima with respect to the $2 k_{g}$ component of the grating profile, with the higher energy band edge having its surface charge density maxima at the troughs of the $2 k_{g}$ component, and the lower energy band edge having its surface charge density maxima located at the peaks of the $2 k_{g}$ component. ${ }^{21}$ Depending on the relative phase of the $2 k_{g}$ component with respect to the fundamental $k_{g}$ component the high-energy band edge can be either the symmetric or antisymmetric surface charge density distribution relative to the fundamental $k_{g}$ component of the grating profile (for a nonblazed grating profile).

If the Fourier series description of the grating profile is a sine series then for an unblazed grating the phase of the $2 k_{g}$ component must be $\pm 90^{\circ}$ with respect to the fundamental $k_{g}$ 

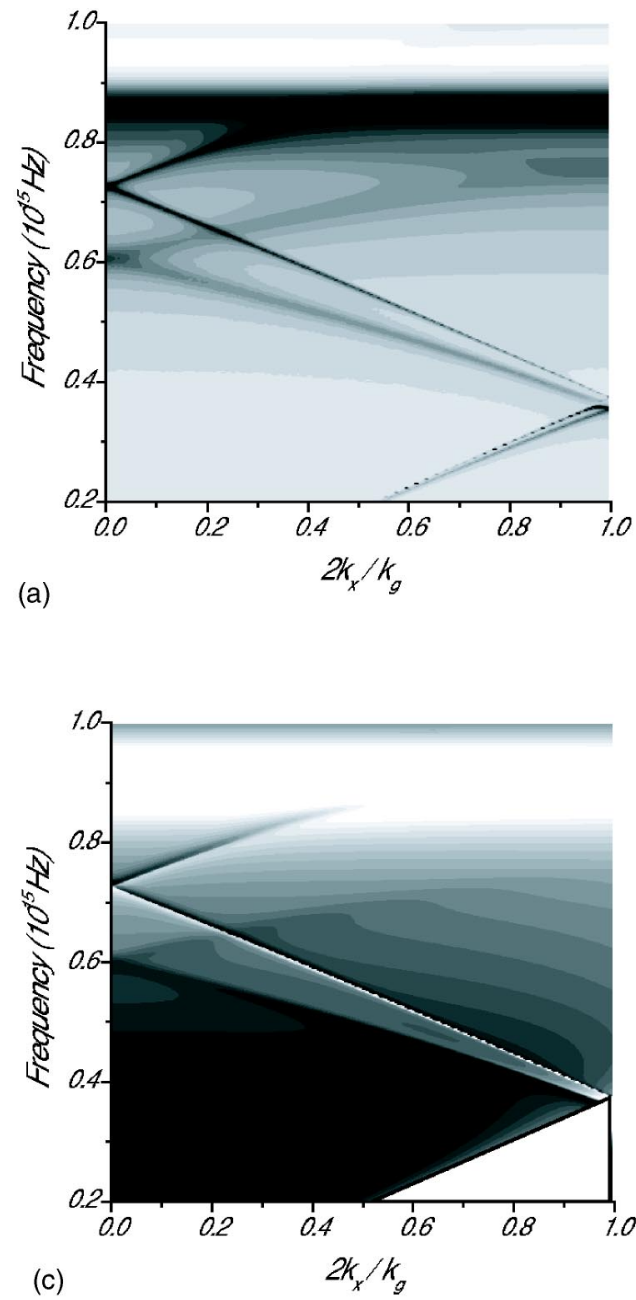
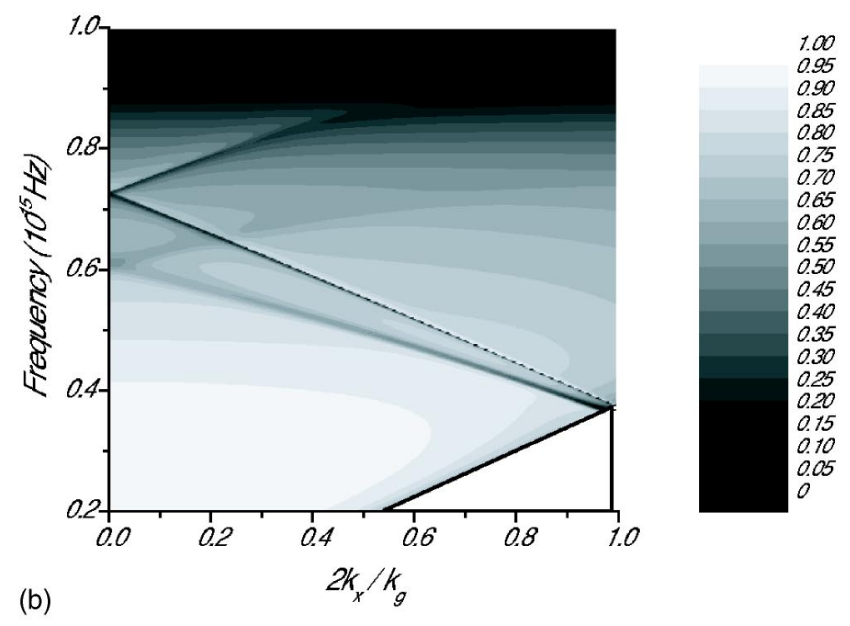

(b)
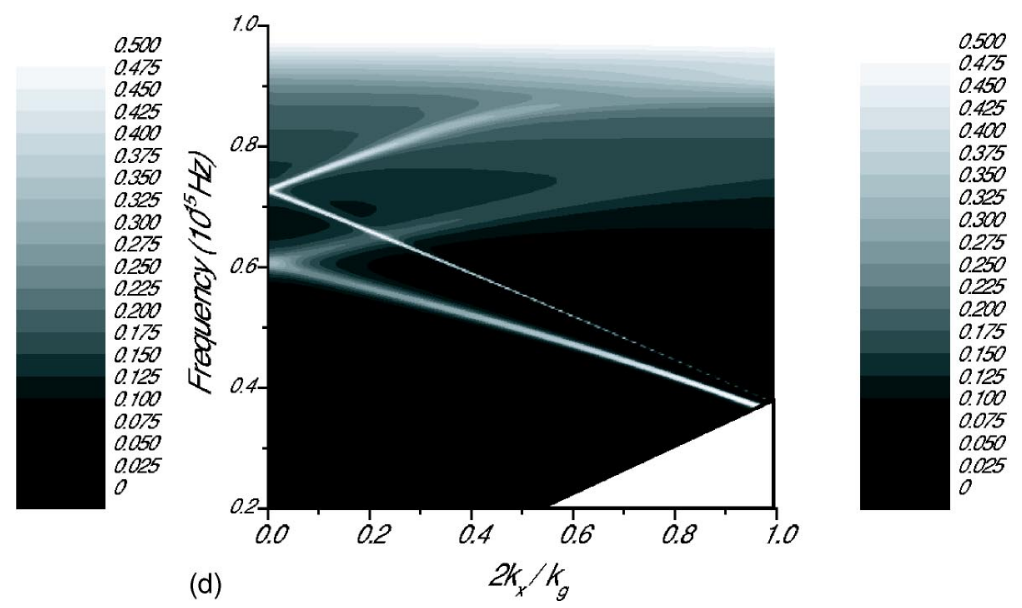

FIG. 2. The optical response of the same system as described for Fig. 1 (the film thickness is $30 \mathrm{~nm}$ ) as a function of frequency and in-plane wave vector. (a) The dispersion of the modes obtained from the scattering matrices, (b) the reflection, (c) the transmission, and (d) the absorption of the system.

component. If the $2 k_{g}$ component is $+90^{\circ}$ out of phase with the $k_{g}$ component the high-energy solution is the symmetric surface charge density distribution (on either side of the fundamental $k_{g}$ component grating peaks), whereas the lowenergy solution is the antisymmetric surface charge density distribution (on either side of the fundamental $k_{g}$ component grating peaks). The reverse is the case if the $2 k_{g}$ component is $-90^{\circ}$ out of phase with the $k_{g}$ component. It is only the antisymmetric surface charge density distribution which may couple to the incident light (due to the orientation of the E-field of the normally incident light with respect to the surface charge densities of the SPP), and therefore only one of the band edges may be coupled to in each case.

For an optically thin metal film (where the thickness is less than approximately $80 \mathrm{~nm}$ ) these band gaps are also opened in the coupled SPP dispersion curves. In Fig. 3 similar plots to those in Fig. 1 are shown, but in this case there is a $5 \mathrm{~nm}$ amplitude $2 k_{g}$ component $+90^{\circ}$ out of phase with the fundamental $k_{g}$ component (of amplitude $10 \mathrm{~nm}$ ) in the grating profiles (the profile of both surfaces is the same as the top interface shown in Fig. 7.

In the case of a purely sinusoidal grating profile on a thick metal slab there is a single SPP mode, which is identical to the single interface SPP. The band-splitting associated with the $2 k_{g}$ component results in two bands for large slab thickness in Fig. 3(a). It must be noted here that standing wave states only arise when $k_{x}=N k_{g} / 2$ (for example at normal incidence, as considered here). Between these points the SPP on a single interface structure is not a standing wave and so the surface charge density maxima are no longer confined to specific regions of the grating profile. The SPP dispersion curve on the single interface structure has fields with a periodicity of $\lambda_{g} / 2$ at the lowest frequency crossing point at normal incidence, with subsequent higher frequency normal incidence crossing points having periodicity of $\lambda_{g} / 2 n$, where $n$ is an integer corresponding to which crossing point is being considered (for example the second lowest frequency normal incidence crossing point has a periodicity of $\left.\lambda_{g} / 4\right)$. At the first Brillouin zone (BZ) boundary (at $k_{x}=k_{g} / 2$ ) the lowest frequency crossing point has field with a periodicity of $\lambda_{g}$, with subsequent higher frequency crossing points having a periodicity of $\lambda_{g} /(2 n-1)$. In regions between these BZ boundaries the fields of the SPP change their periodicity between that occurring at one BZ boundary and that at the other. When considering the thin slab structure the two coupled SPP solutions (the LRSPP and SRSPP) have the 

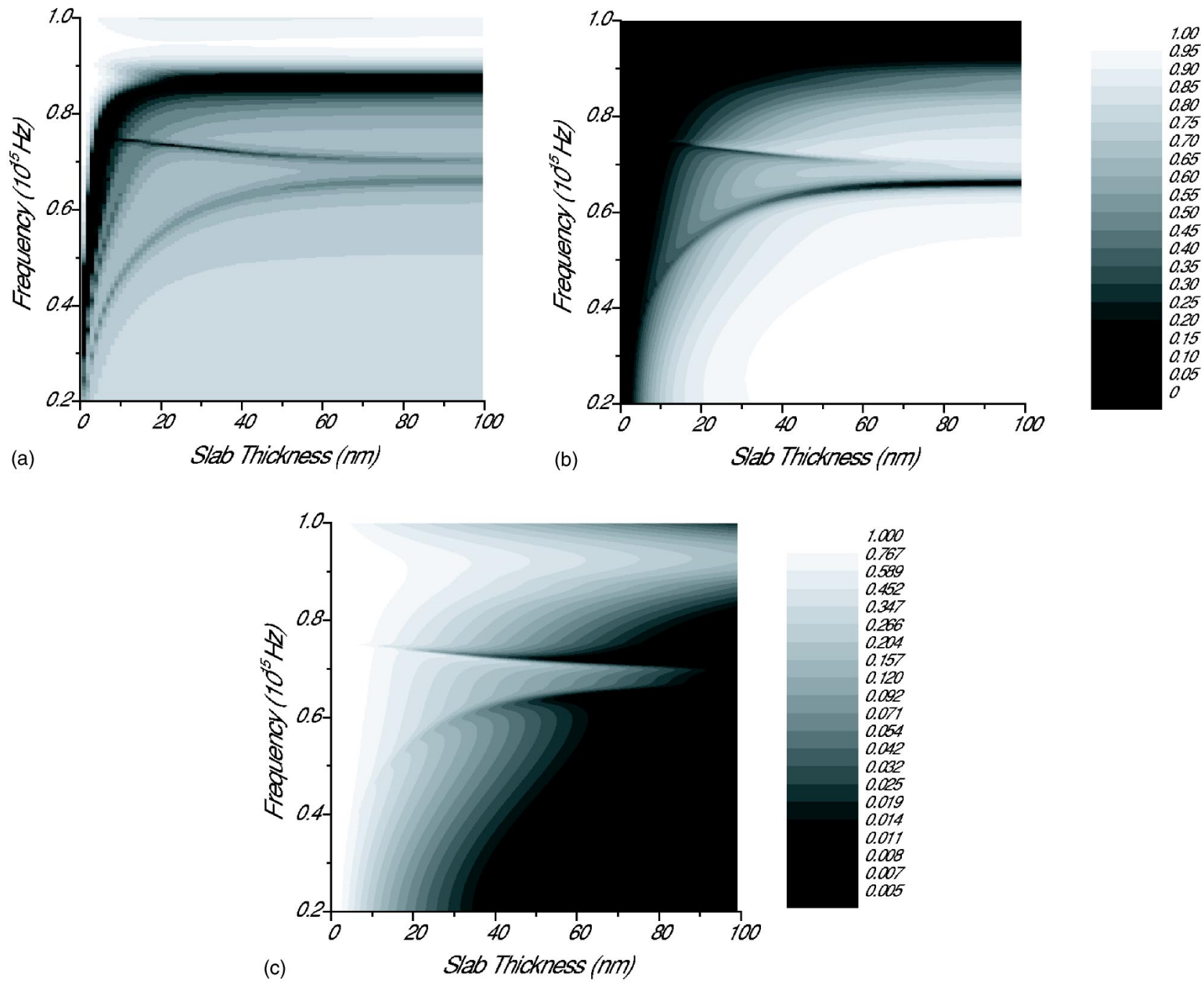

FIG. 3. The optical response of the same system as Fig. 1 , but with an additional $5 \mathrm{~nm}$ amplitude $2 k_{g}$ component (+90 out of phase with the $k_{g}$ component) in the grating profile description, as a function of frequency and slab thickness. (a) The position of the modes in frequency obtained from the scattering matrices, (b) the reflectivity, and (c) the transmissivity (log scale) of the system.

same periodicities at the BZ boundaries as do the SPPs on a single interface structure. Similarly their periodicities change in the region between the BZ boundaries where they are no longer standing wave modes. Therefore, the arguments concerning the surface charge density distributions of the standing wave modes at normal incidence are not valid for finite values of $k_{x}$. However, since the dispersion of the coupled SPPs varies smoothly between the two BZ boundaries at $k_{x}$ $=0$ and $k_{x}=k_{g} / 2$ the frequency at the BZ boundaries of the two coupled modes is clearly evidenced at all values of $k_{x}$.

From Fig. 3 it is apparent that at lower slab thickness the LRSPP and SRSPP evolve directly from the high and lowenergy edges of the band gap, respectively. This is a somewhat surprising evolution since the positions of the surface charge maxima on the upper surface for the SRSPP and LRSPP are identical, whereas those of the high and lowenergy band edges are not.

To understand why these modes evolve as they do it is useful to investigate the dispersion curves of the SPPs of the system for different slab thickness. This is shown in Fig. 4, where the dispersion curves are shown for slab thickness of $70 \mathrm{~nm}, 50 \mathrm{~nm}$, and $30 \mathrm{~nm}$.

From Fig. 4 it is clear that, for a $70 \mathrm{~nm}$ thick metal slab, there is anticrossing between the LRSPP and the SRSPP very close to the position of the band gap. In fact, because this anticrossing is so close to the band gap, the modes around this point must be considered as having mixed SRSPP and LRSPP character. For decreasing slab thickness the anticrossing occurs further from the $k_{x}=0$ axis, so that the two modes at $k_{x}=0$ become more LRSPP and SRSPP in character the thinner the slab becomes. This leads to the conclusion that, when the slab thickness is made large enough such that the SRSPP and LRSPP overlie each other, the anticrossing occurs at the position of the band gap and that in this case the band gap and anticrossing processes are the same.

For a single interface grating with a $2 k_{g}$ component the coupling to the modes for normally incident light depends upon the phase of the $2 k_{g}$ component with respect to the $k_{g}$ component, with coupling only possible to the mode with an antisymmetric charge distribution on either side of a grating 

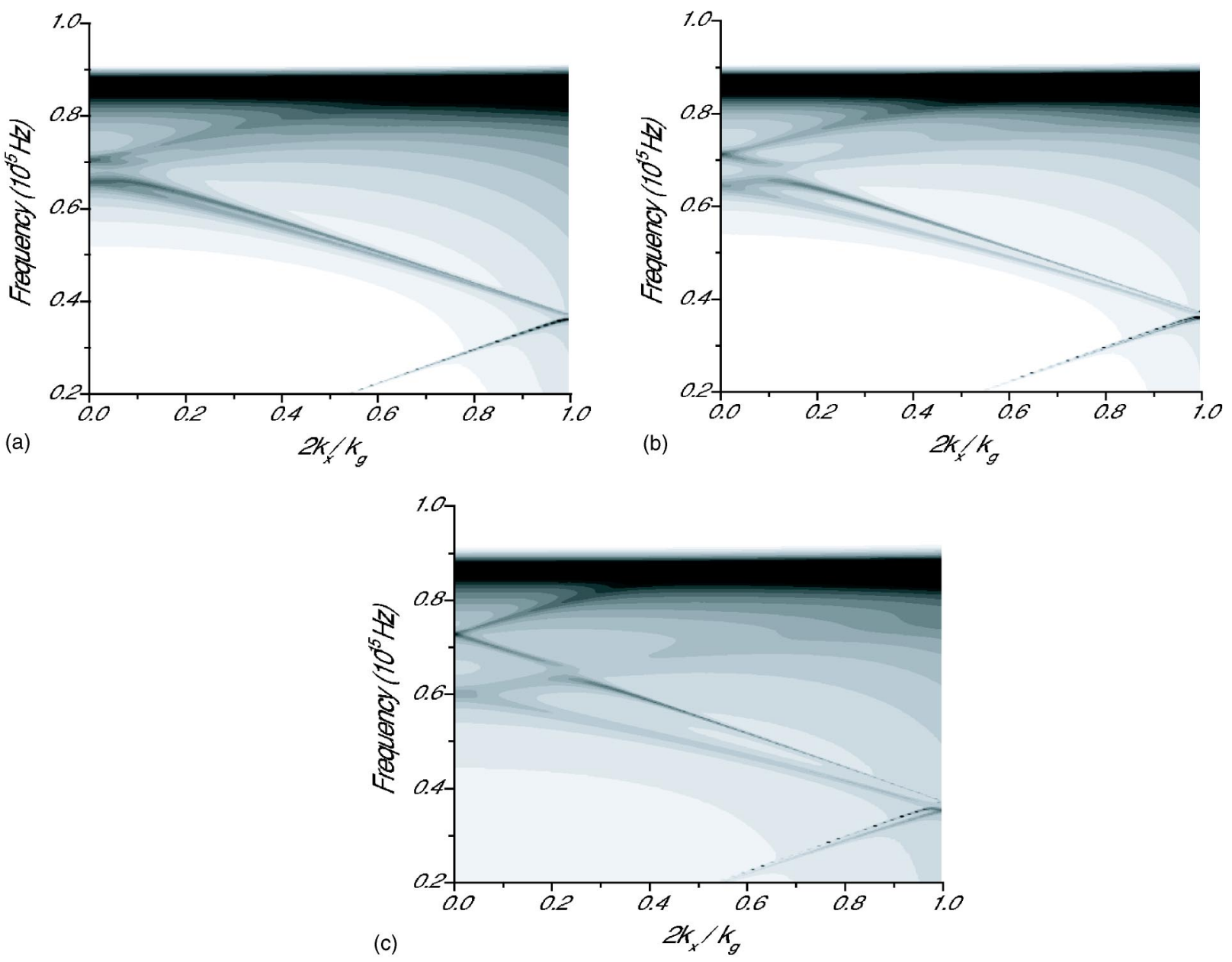

FIG. 4. The band structure of the SPPs of the system (obtained from the scattering matrices) for the same structure as for Fig. 3 but for different slab thickness. (a) $d=70 \mathrm{~nm}$, (b) $d=50 \mathrm{~nm}$, and (c) $d=30 \mathrm{~nm}$.

peak. The same is true of the coupled SPP modes, and therefore, when the grating slab thickness is relatively large and the coupled SPP modes are largely similar to the single interface SPP, only one band edge is coupled to. As the layer thickness is decreased, so that the modes become more LRSPP and SRSPP like, coupling to both modes becomes possible.

The reason that both standing wave modes can be coupled to at normal incidence for lower slab thickness is that the two solutions for the two coupled LRSPPs and SRSPPs (the symmetric and antisymmetric surface charge distributions on either side of the peaks) overlie each other in energy (and therefore frequency). This overlying in energy arises because the light on the incident side "sees" a $2 k_{g}$ component with a $+90^{\circ}$ phase difference with respect to the $k_{g}$ component, whereas the light transmitted through the structure "sees" a $2 k_{g}$ component with a $-90^{\circ}$ phase difference with respect to the $k_{g}$ component. For the standing wave modes excited at normal incidence on this system the charge distributions on the two sides of the structure occur in the troughs of the $2 k_{g}$ component on one surface, and on the peaks of the $2 k_{g}$ component on the other surface. This is clearly shown in Fig. 5, where the $H_{z}$ component of the fields (the $z$ direction is into the page) of the two modes which may be coupled to at normal incidence are shown for the case shown in Fig. 3, for a slab thickness of $30 \mathrm{~nm}$. The positions of maximum surface charge density occur at the minimum positions of $H_{z}$ (on the surface) since the $H$ and $E$ fields of the SPPs are $90^{\circ}$ out of phase with each other. Therefore, since these occur on the sides of the grating grooves, the positions of maximum surface charge density on the top surface occur at the peaks of the $2 k_{g}$ component and the positions of maximum surface charge density on the bottom surface occur at the troughs of the $2 k_{g}$ component. Since both possible solutions for the two coupled modes will experience the same influence from the $2 k_{g}$ component of the grating profile, and any energy difference between them will be due to the $2 k_{g}$ component, both LRSPP modes and both SRSPP modes will have the same energy. The higher energy mode in Fig. 3 would, therefore, be expected to show no coupling for large slab thickness [as is seen in Fig. 3(b)], but would show coupling for lower slab thickness. The opposite is true if the $2 k_{g}$ component is $-90^{\circ}$ out of phase with the $k_{g}$ component, and the LRSPP could be coupled to for any slab thickness (noting that the describer LRSPP becomes meaningless beyond a slab thickness of approximately $80 \mathrm{~nm}$, since the nature of the mode changes with increased thickness to become the single interface SPP band edge), whereas the SRSPP could only be coupled to for lower slab thickness. 

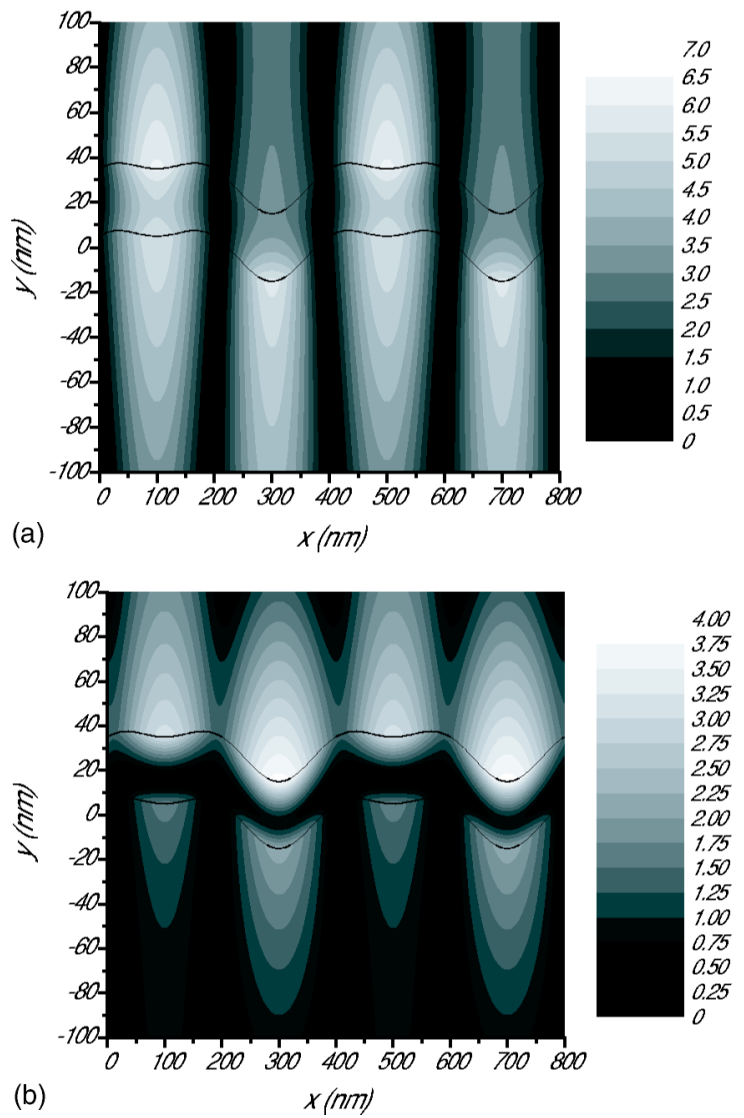

FIG. 5. $H_{z}$ profiles for normal incidence of the long range (a) and short range (b) surface plasmon modes which can be excited on a conformal grating structure with a $2 k_{g}$ component. The modes correspond to those shown in Fig. 3, with a metal slab thickness of $30 \mathrm{~nm}$.

\section{COUPLED SPPS ON THIN METAL SLABS IN A NONCONFORMAL GEOMETRY}

\section{A. Sinusoidal corrugations}

In this section the case of a thin metal slab bounded by dielectrics with identical dielectric functions (in this case air), and corrugated with grating structures on both surfaces, but with a phase difference between the two gratings is considered. Particular attention is paid to the case where the lower corrugation (on the transmission side of the structure) is in antiphase with the upper corrugation (on the incident side of the structure).

The case where the two corrugations are perfectly sinusoidal is initially considered. In Fig. 6 the zeroth order reflectivity, transmissivity, and absorption of the system are shown for a $30 \mathrm{~nm}$ thick silver slab corrugated on both surfaces by a $400 \mathrm{~nm}$ pitch, $10 \mathrm{~nm}$ amplitude, sinusoidal grating, as a function of frequency and phase difference between the corrugations on the two interfaces.

Before considering the optical response of the system shown in Fig. 6 it is useful to examine the absorption. The maximum in the absorption occurs at the frequency at which the coupled SPPs are excited. It is clear from Fig. 6(c) that the excitation frequency of the SRSPP reduces as the phase difference between the corrugations on each surface is in- creased. Also to be noted is the change in coupling strength to the modes as this phase difference is changed.

In order to understand the reduction in the excitation frequency of the SRSPP as a function of the phase difference between the corrugations, it is necessary to consider the effective thickness of silver which the evanescently decaying fields of the SPPs experience. For the conformal structure the thickness of the silver film is constant across a period of the grating and hence the surface charges on the top surface always experience the same thickness of silver. However, for the antiphase structure the surface charges near the troughs experience a smaller thickness of silver than those towards the peaks. Due to the fact that the fields associated with the surface charge density distribution on the top surface exponentially decay through the silver film, the resulting effect is that for the antiphase structure the total field at the bottom surface due to the overall surface charge density distribution at the top surface is greater than that for the conformally corrugated structure. Therefore if, as is the case here, the corrugation amplitude is of the order of the film thickness, the SRSPP experiences an effectively thinner silver film when the corrugations are in antiphase. Figure 1(c) shows that the frequency at which the SRSPP on a conformal structure is excited reduces with the silver film thickness. Since, for the antiphase structure, the SRSPP experiences an effectively thinner silver film thickness, it is excited at a lower frequency than for the conformally corrugated structure.

The change in the coupling strength to the two coupled SPP modes as a function of the phase difference between the corrugations on the two surfaces can best be understood by considering a simple analogy to the coupled SPP system. If two pendulums are connected together with a spring and one is driven harmonically there are two possible steady state solutions: one in which the two pendulums oscillate in phase, and one in which they oscillate out of phase. These two solutions are analogous to the SRSPP and LRSPP on the thin metal slab systems, except that in this case the system is more complicated since the charge distributions on either side of the metal slab (the two pendulums in the analogy) can both be driven (by the reflected and transmitted diffracted orders). The total transmitted diffracted order field of a corrugated thin metal slab in a conformal geometry is nearly zero since the transmitted diffracted orders created at the two interfaces cancel. ${ }^{8}$ Therefore, the conformal geometry corresponds to the case where the charge distribution on only the incident interface is driven. When the phase of the bottom surface corrugation is changed with respect to the corrugation on the top (incident) surface the total transmitted diffracted order fields are no longer zero, resulting in the charge distributions on both interfaces being driven. The total transmitted diffracted order fields are at a maximum at a phase difference between the two corrugations of approximately $180^{\circ}$ (with some small change due to the thickness of the metal slab).

The coupling strength of the incident light to the SRSPP increases with increasing phase difference between the two corrugations, while the coupling strength to the LRSPP decreases. This can be explained by considering the phase of the transmitted diffracted order with respect to the charge oscillations of the coupled SPPs on the bottom interface. 

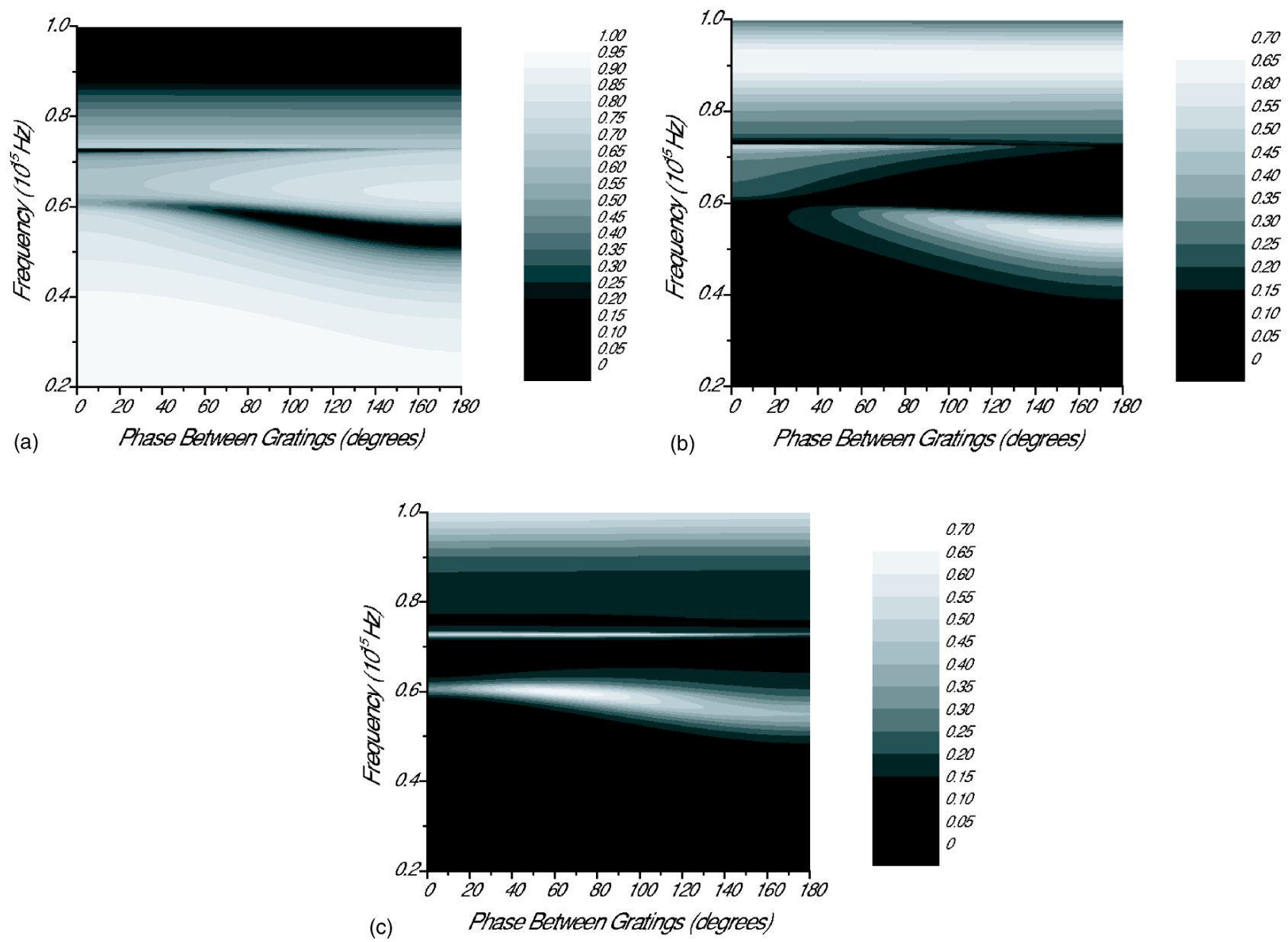

(c)

FIG. 6. The zeroth order normal incidence reflectivity (a), transmissivity (b), and absorption of the system (c), for a $30 \mathrm{~nm}$ thick silver slab corrugated on both surfaces with a $400 \mathrm{~nm}$ pitch, $10 \mathrm{~nm}$ amplitude, sinusoid as a function of frequency and phase between the corrugations on the two surfaces.

This is analogous to the phase of the driving force on the second pendulum being different to the phase of acceleration of the second pendulum. If the driving force on the second pendulum is in phase with the acceleration the coupling strength will be increased, whereas if it is out of phase the coupling strength will be decreased. Since the charge distributions on the bottom interface of the thin metal slab for the SRSPP and LRSPP are out of phase with each other this means that the coupling strength to one of the modes will be increased with an increase in the transmitted diffracted order, whereas the coupling strength to the other mode mode will be reduced.

\section{B. Sinusoidal corrugations with an additional $2 k_{g}$ component}

The effect of adding a $2 k_{g}$ component to the grating profile will now be considered for an anti-symmetric corrugated structure (the mirror plane occurring at the center of the thin slab). The modelling presented is for the case where the $2 k_{g}$ component on the top interface is $+90^{\circ}$ out of phase with the $k_{g}$ component (the differences in the optical response of the structure when the phase is $-90^{\circ}$ are also explained). Since it is an antisymmetric structure that is of interest this requires the $2 k_{g}$ component for the lower interface to be $180^{\circ}$ out of phase with the $2 k_{g}$ component on the top interface. The resulting structure is shown in Fig. 7. These structures are similar to those studied by Tan et al. ${ }^{22}$ in which a silver film was corrugated on both surfaces by Gaussian grooves of opposite signs. Very high transmission was found to occur in this case.

A plot of the band structure, and of the reflectivity and transmissivity of the zeroth order for normally incident light from the structure schematically shown in Fig. 7 as a function of frequency and slab thickness is shown in Fig. 8 (the gratings have a $10 \mathrm{~nm}$ amplitude $k_{g}$ component, a $5 \mathrm{~nm} 2 k_{g}$ component, and a pitch of $400 \mathrm{~nm}$ ).

It is clear from Fig. 8(a) that the dispersion of the modes is very different to that of the conformal case having a $2 k_{g}$ component (Fig. 4). In that case there were two modes evident: one which corresponded to the LRSPP (which developed from the high-energy branch of the SPP dispersion curve for large slab thickness), and one which corresponded to the SRSPP (which developed from the low-energy branch of the SPP dispersion curve for large slab thickness). In Fig. 7 , however, there are now four different modes evident (in fact for low slab thickness there are five since the second order SRSPP has reduced in frequency to just below the two 


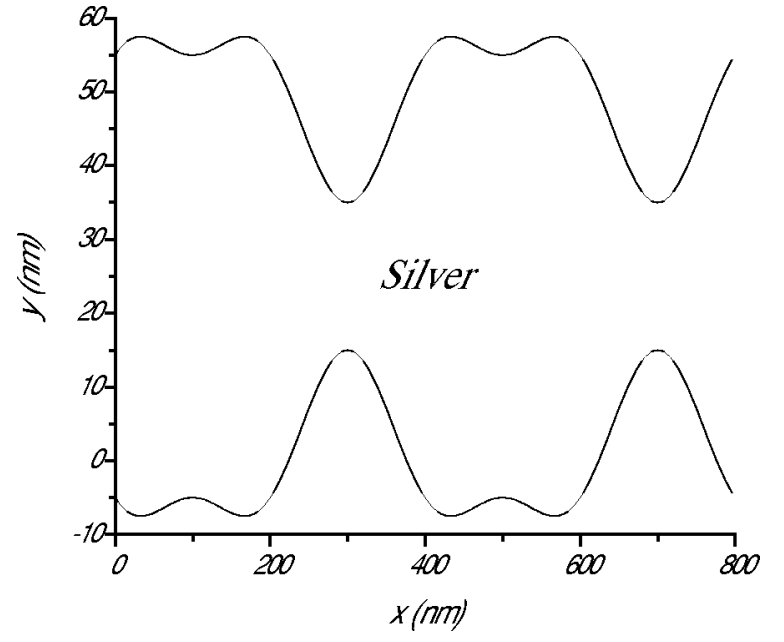

FIG. 7. Schematic of the structure being investigated. There is a $10 \mathrm{~nm}$ amplitude $k_{g}$ component, and a $5 \mathrm{~nm}$ amplitude $2 k_{g}$ component. The grating pitch is $400 \mathrm{~nm}$. In this case the average film thickness is $50 \mathrm{~nm}$. first order LRSPPs). Two of these correspond to LRSPPs and two to SRSPPs, and these develop from both the high and low-energy band edges of the band gap of the SPP dispersion curve at large slab thickness.

In the conformal case the two LRSPP solutions, and the two SRSPP solutions, had the same energy, and therefore they overlaid each other in the plots of Fig. 4. For this new structure this is no longer the case since the surface charge distributions of the standing wave LRSPP and SRSPP with their maximum surface charge densities at the maxima and minima of the $k_{g}$ component of the grating profile have a different thickness of metal between them than do the solutions with maximum surface charge densities at the midpoints between the maxima and minima of the $k_{g}$ component. Therefore, the two LRSPP and SRSPP modes have different energies and occur at different frequencies for low slab thickness. Schematic diagrams of the distributions of the surface charge density maxima for these four possible standing wave modes (for normal incidence) are shown in Fig. 9.

The $H_{z}$ component of the fields of these four modes for the structure shown in Fig. 7 with a slab thickness of $70 \mathrm{~nm}$ are shown in Fig. 10, with the thickness chosen so that all four modes could be excited, and distinguished from one another, at near normal incidence. As with the field profiles
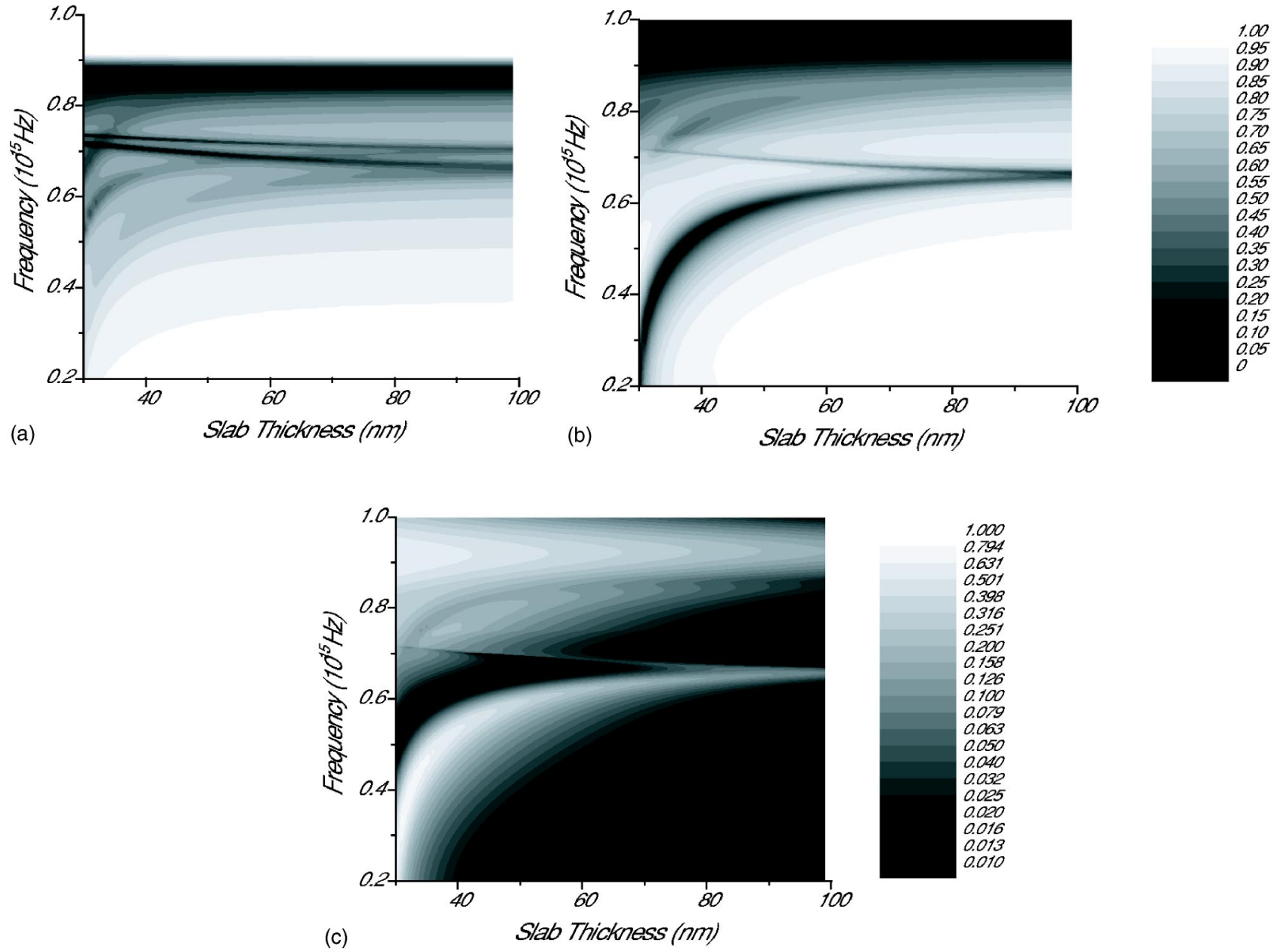

FIG. 8. The band structure (a), reflectivity (b), and transmissivity (c) (log scale) of the zeroth order, for normally incident light as a function of frequency and slab thickness for the structure shown in Fig. 7. 

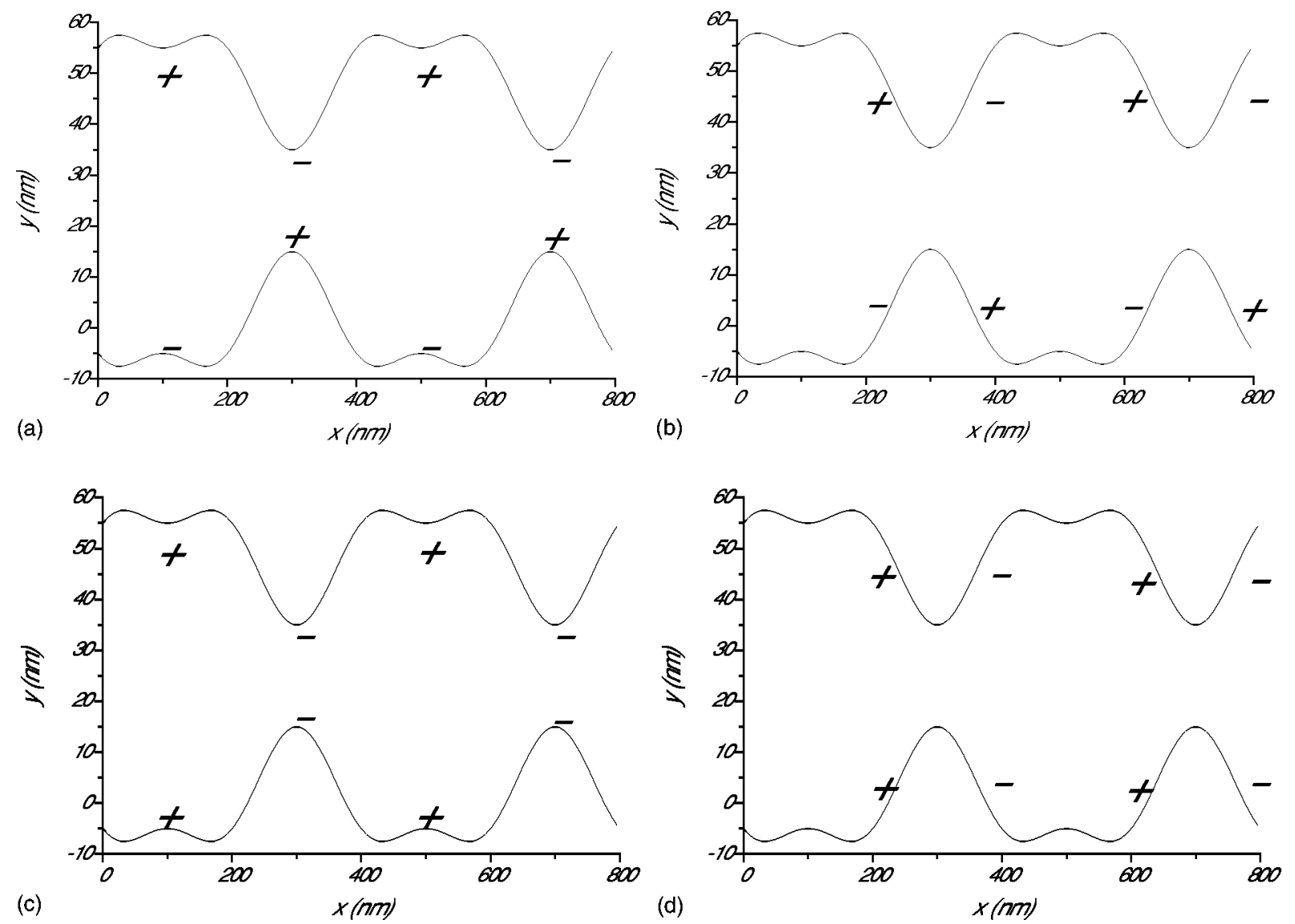

FIG. 9. Schematics showing the positions of maximum surface charge density of the four possible standing wave coupled SPP modes for normal incidence that may be excited on the structure. (a) The LRSPP from the high energy band edge, (b) the LRSPP from the low energy band edge, (c) the SRSPP from the high energy band edge, and (d) the SRSPP from the low energy band edge.

presented in Fig. 5 the positions of the surface charge density maxima occur at the points on the surface where the $H_{z}$ component has its minima. Since only the two of these modes which have their surface charge density maxima on the sides of the grating ridges can be coupled to at normal incidence the field profiles have been generated for an incident wave vector of $2 k_{x} / k_{g}=0.05$. It is clear that the two field profiles corresponding to the modes with their surface charge density maxima on the sides of the grating ridges [Figs. 10 (b) and 10(d)] behave as would be expected, with the long range mode having stronger coupling through the metal film than the short range mode. The two modes which have their surface charge density maxima at the peaks and troughs of the structure [Figs. 10(a) and 10(c)] have very different field profiles on their two interfaces. The field distribution on the bottom interface is as would be expected from the schematic of the surface charge density maxima shown in Fig. 9, however on the top surface the fields are significantly perturbed because the mode may only be coupled to at nonzero angles of incidence, nevertheless the stronger coupling of the long range mode through the metal slab is still evident.

Whereas on the conformal structure the two short range SPPs develop from the same band edge, on this structure one short range SPP develops from the low energy band edge and one develops from the high energy band edge. The same is true of the two long range SPP modes. The surface charge density distribution of the coupled SPP mode on the top (incident) surface corrugation is excited by the reflected diffracted order and "sees" a $2 k_{g}$ component which is $+90^{\circ}$ out of phase with the $k_{g}$ component. The bottom surface charge density of the coupled SPP mode is excited by the transmitted diffracted order which, even though the $2 k_{g}$ component of the grating is $-90^{\circ}$ out of phase with the $k_{g}$ component, "sees" the $2 k_{g}$ component as being $+90^{\circ}$ out of phase (since the transmitted diffracted order becomes evanescent from the transmission side of the structure it experiences an identical surface as does the reflected diffracted order on the reflection side of the structure). The standing wave LRSPP with surface charge density maxima on both surfaces occurring at the peaks and troughs of the $k_{g}$ component has its electric field maxima at the troughs of the $2 k_{g}$ component on both surfaces. For a single interface grating this would correspond to the high-energy band edge of the band gap at normal incidence. Correspondingly, the LRSPP with surface charge density maxima on both surfaces at the midpoints between the maxima and minima of the $k_{g}$ component has its electric field maxima at the peaks of the $2 k_{g}$ component on both surfaces, and this would correspond to the low-energy band edge of the band gap at normal incidence for a single interface grating. Therefore, it is apparent that the two possible LRSPP modes develop from the two band edges of the band gap as the slab thickness is reduced. The same arguments are true for the two SRSPP modes. 

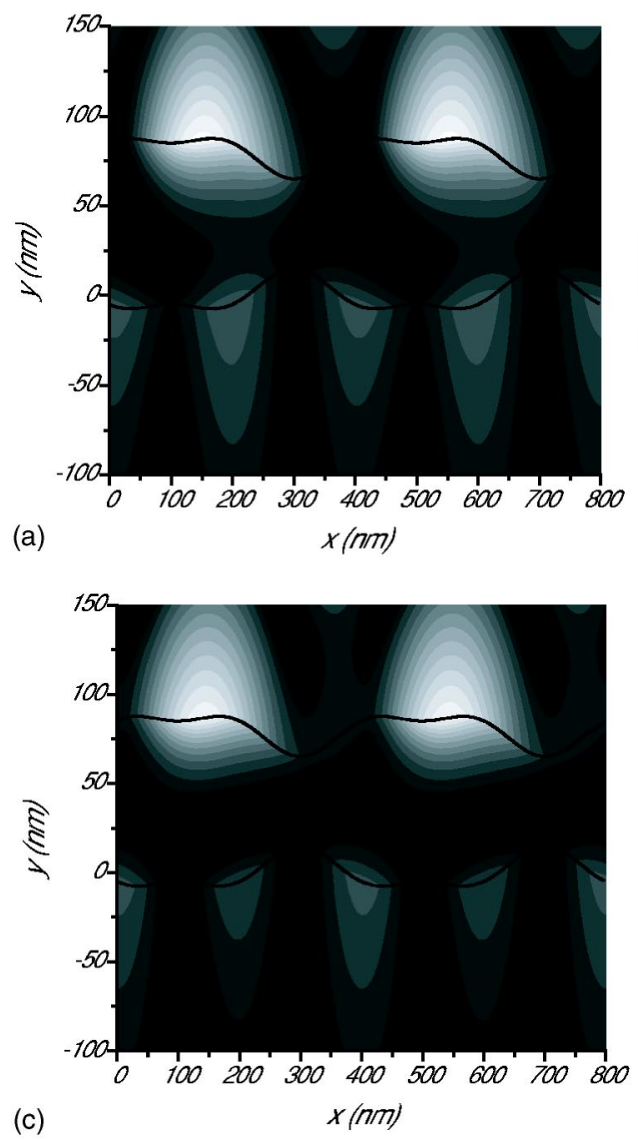
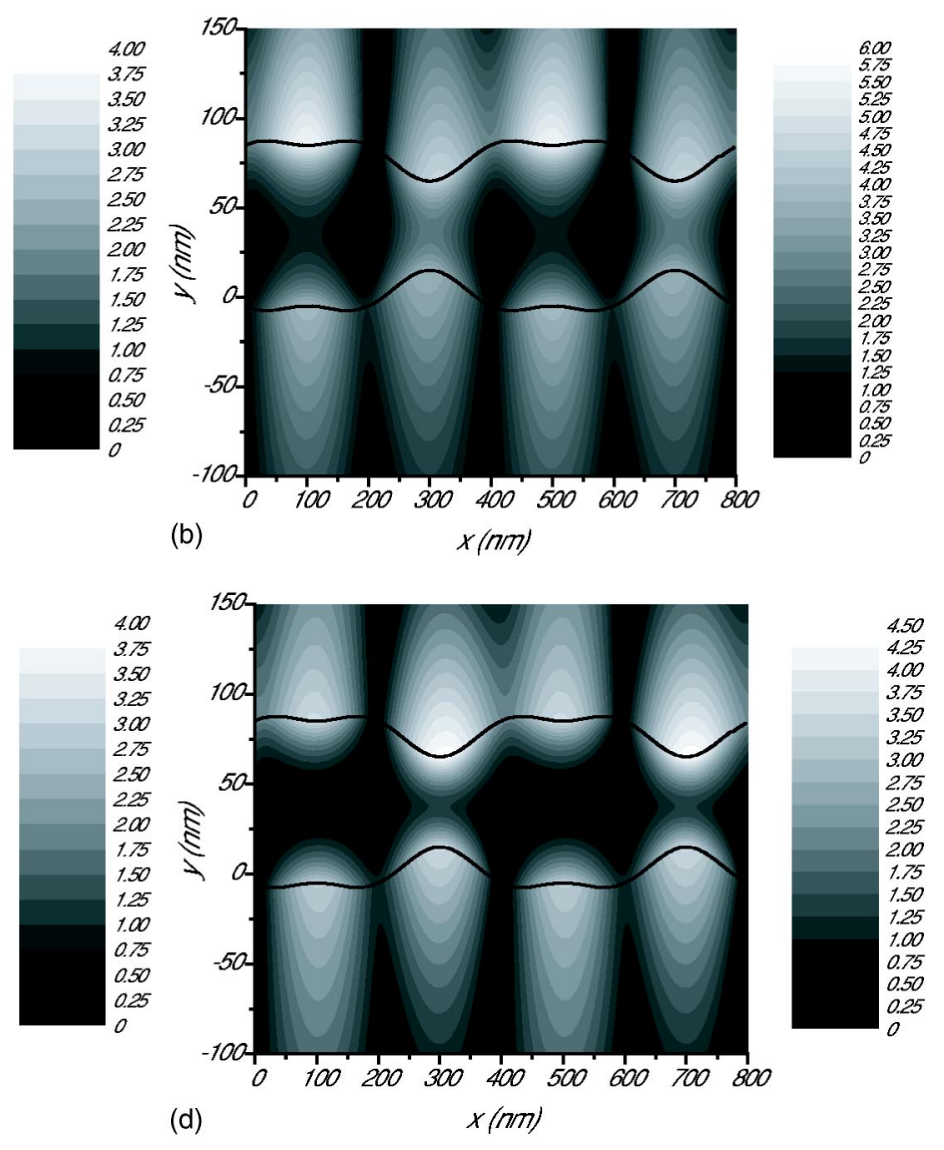

FIG. 10. Field profiles $\left(H_{z}\right.$ where $z$ is into the page) of the four possible first order SPP modes excitable on the structure shown in Fig. 7, but with an average thickness of $80 \mathrm{~nm}$, that can be excited on this structure for $2 k_{x} / k_{z}=0.05$. (a) The LRSPP developing from the high energy band edge, (b) the LRSPP developing from the low energy band edge, (c) the SRSPP developing from the high energy band edge, and (d) the SRSPP developing from the low energy band edge.

It is clear from the reflectivity and transmissivity plots in Fig. 8 that coupling only occurs to the LRSPP and SRSPP developing from the low-energy band edge at large slab thickness (with the shape of the resonance features having the same forms as those described in the previous section for antiphase sinusoidally corrugated systems). This is because it is only the standing wave modes which have their surface charge density maxima at the midpoints between the maxima and minima of the $k_{g}$ component of the grating profile which may be coupled to.

If the phase of the $2 k_{g}$ component on the top surface (remembering that the $2 k_{g}$ component on the bottom surface has the opposite sign) is $-90^{\circ}$ rather than $+90^{\circ}$, then the LRSPP and SRSPP modes developing from the high-energy band edge at large slab thickness will be coupled to rather than those from the low-energy band edge. If the phase is $0^{\circ}$ then all four modes will be coupled.

In Fig. 11 the zero-order reflectivity as a function of frequency and in-plane wave vector is presented for the same structure (shown in Fig. 7), for four different film thicknesses: $80 \mathrm{~nm}$ (a), $50 \mathrm{~nm}$ (b), $40 \mathrm{~nm}$ (c), and $30 \mathrm{~nm}$ (d).

In Fig. 11(a) there is only a small splitting of the SPP dispersion curves into the LRSPP and SRSPP. Due to the phase of the $2 k_{g}$ component of the grating profile with respect to the fundamental $k_{g}$ component it is only the lower energy LRSPP and SRSPP which are coupled to for normally incident light $\left(k_{x}=0\right)$. As the thickness of the film is reduced [Figs. 11(b)-11(d)] the SRSPP moves away from the (diffracted) light line and the LRSPP moves toward the (diffracted) light line.

An interesting point to note is the different rate at which the two SRSPPs (originating from the high and low-energy band edges) move away from the (diffracted) light line for normally incident light. The SRSPP which develops from the low-energy band edge reduces in frequency more rapidly than the SRSPP which develops from the high-energy band edge. The same is true for the SRSPPs occurring at the Brillouin zone boundary at $k_{x}=k_{g} / 2$. This is due to the fact that, for the phase difference between the $k_{g}$ and $2 k_{g}$ components studied here, the SRSPP developing from the low energy band edge has its surface charge density maxima at the peaks and troughs of the structure, where the exponentially decaying fields within the metal experience an effectively much thinner metal film than does the SRSPP developing from the high energy band edge which has its surface charge density maxima on the sides of the grooves. The different rate at which the excitation frequencies of the two SRSPPs (which develop from the two band edges) reduce causes the SRSPP 

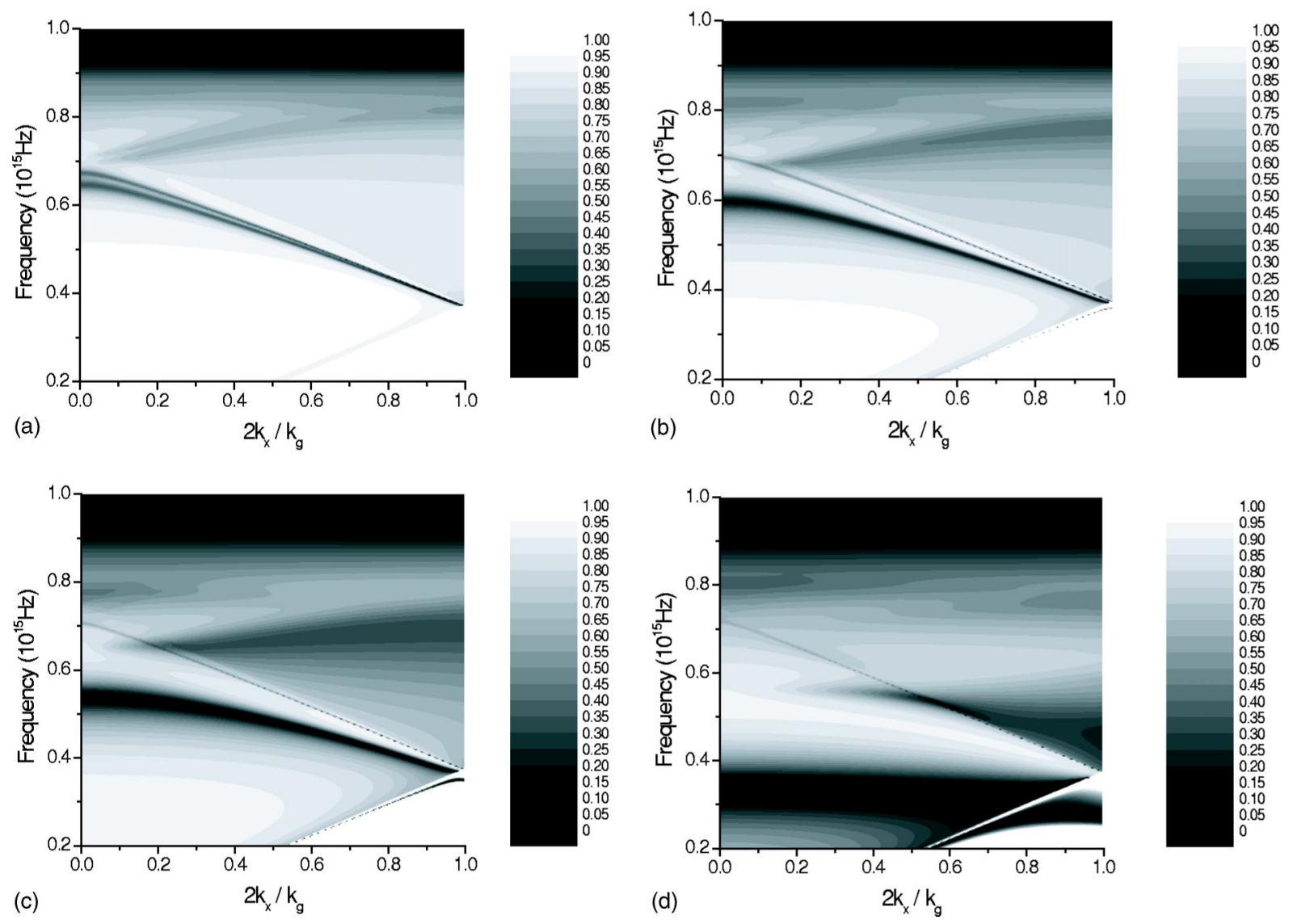

FIG. 11. The reflectivity as a function of frequency and in-plane wave vector for the same system described for Fig. 7, and for film thickness of (a) $80 \mathrm{~nm}$, (b) $50 \mathrm{~nm}$, (c) $40 \mathrm{~nm}$, and (d) $30 \mathrm{~nm}$.

which develops from the low-energy band edge at normal incidence to reduce in frequency more rapidly than the SRSPP which develops from the high-energy band edge at the Brillouin zone boundary. This is the cause of the very flat-banded reflectivity minimum evident in Fig. 11(a).

In Fig. 12 similar plots to those in Fig. 11 are shown, but in this case it is the transmission rather than the reflectivity which is plotted. The dispersion of these modes is the same as for those in Fig. 11, but transmission maxima are now evident when the SPPs are excited. In the case of the $30 \mathrm{~nm}$ thick film [Fig. 11(d)] this has resulted in a very flat and strong $(>80 \%)$ transmission band. This is much stronger than the transmission through the conformally corrugated structures. It is important to note that, since this transmission band is extremely flat over the full range of possible in-plane wave vectors, the same frequency of light will have its transmission enhanced by the coupled SPPs for all incident angles. However, it must be noted that the flat-banding of the transmission maxima only occurs when the thickness of the silver film at the minima of the grating grooves approaches zero. It should also be noted that since the silver film is effectively thinner than the average thickness (because the strong evanescent fields are located in the grooves of the grating where the thickness is very small) this large transmission may be expected. However, it is clear that this is only true when the SPP mode is excited, and so this increased transmission should only be compared to the transmission off resonance, where the transmission is almost zero. Therefore, the enhancement factor for this transmission due to the excitation of the coupled SPP is very large indeed.

\section{SUMMARY}

In this paper a thin metal slab corrugated on both surfaces and bounded by dielectrics with identical dielectric functions has been investigated. For such a system coupled SPP modes (the LRSPP and SRSPP) can be excited, and their nature, and their effect on the optical response, has been explored for a variety of structures.

Initially, the simple case of a conformally corrugated structure (identical sinusoidal corrugations on the two surfaces) was discussed. Following this it was found that, when a small $2 k_{g}$ component is added to the grating profile, anticrossing between the LRSPP and SRSPP modes occurs in the dispersion curves. With increasing slab thickness this anticrossing becomes the same as the band gaps observed on single interface gratings, with the LRSPP developing from the high-energy band edge, and the SRSPP developing from the low-energy band edge.

The case where the two corrugations on each surface are nonconformal has also been considered. For purely sinusoidal corrugations the coupling strength to the two coupled 

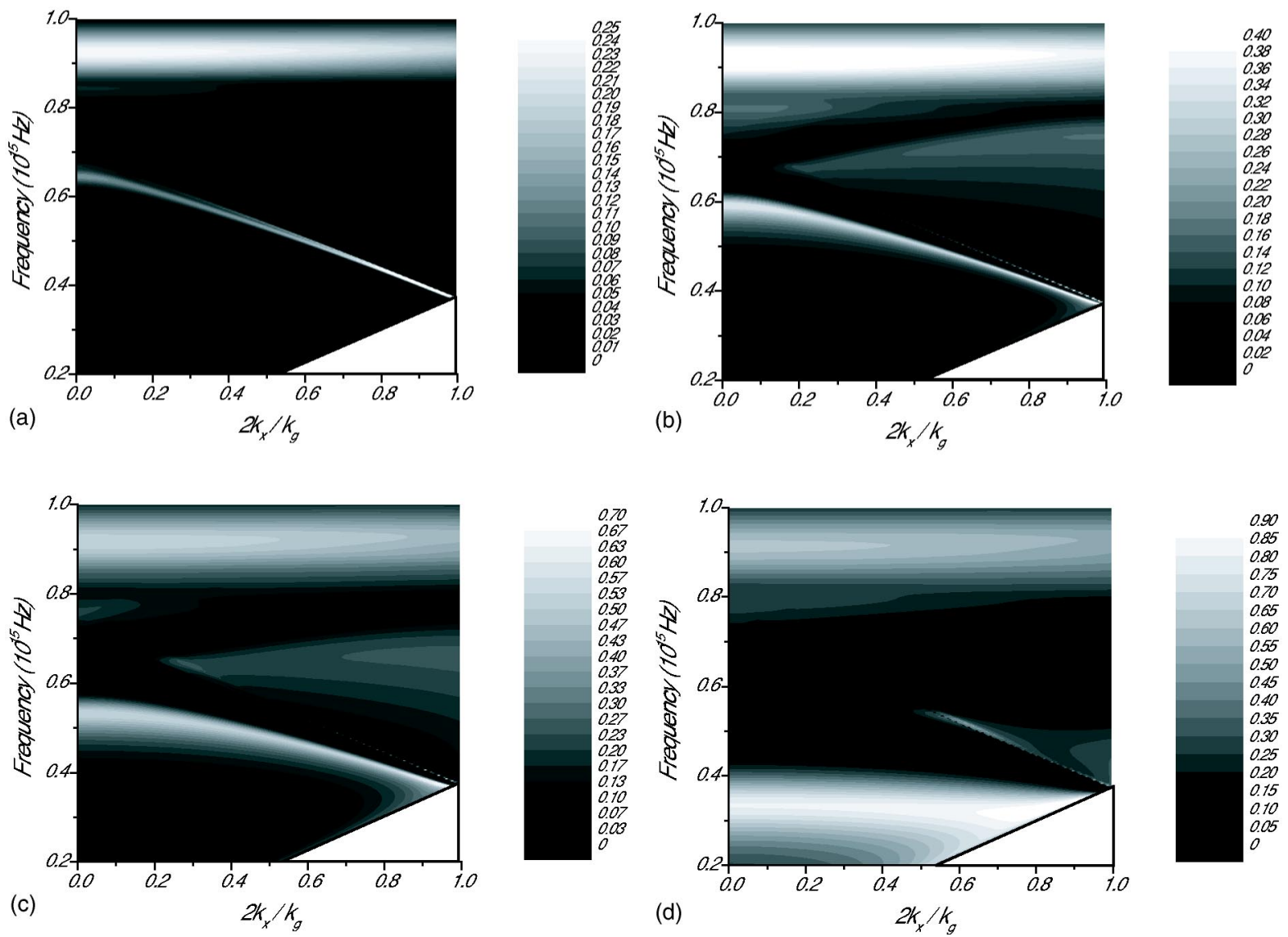

FIG. 12. The transmissivity as a function of frequency and in-plane wave vector for the same system described for Fig. 7, and for film thickness of (a) $80 \mathrm{~nm}$, (b) $50 \mathrm{~nm}$, (c) $40 \mathrm{~nm}$, and (d) $30 \mathrm{~nm}$.

SPP modes alters as a function of the phase difference between the two corrugations. This has been shown to be due to a change in the magnitude of the transmitted diffracted order as a function of this phase difference. The SRSPP mode couples more strongly to the incident radiation with increasing phase difference between the two corrugations, whereas the LRSPP couples more weakly. The effect of adding a $2 k_{g}$ component to the grating surface when the two corrugations are in antiphase is that four different coupled SPP modes exist, with two LRSPP and two SRSPP modes. By investigating the optical response as a function of both frequency and in-plane wave vector it has been shown that in this case a strong transmission $(>80 \%)$ band, which is almost independent of the angle of incidence, can occur due to the excitation of a SRSPP.

\section{ACKNOWLEDGMENTS}

The authors are grateful for EPSRC support and the provision of a CASE award by QinetiQ (Farnborough) for I.R.H. This work was carried out as part of Technology Group 08 of the MoD Corporate Research Fund.
${ }^{1}$ T.W. Ebbesen, H.J. Lezec, H.F. Ghaemi, T. Thio, and P.A. Wolff, Nature (London) 391, 667 (1998).

${ }^{2}$ H.F. Ghaemi, T. Thio, D.E. Grupp, T.W. Ebbesen, and H.J. Lezec, Phys. Rev. B 58, 6779 (1998).

${ }^{3}$ L. Salomon, F.D. Grillot, A.V. Zayats, and F. de Fornel, Phys. Rev. Lett. 86, 1110 (2001).

${ }^{4}$ L. Martin-Moreno, F.J. Garcia-Vidal, H.J. Lezec, K.M. Pellerin, T. Thio, J.B. Pendry, and T.W. Ebbesen, Phys. Rev. Lett. 86, 1114 (2001).

${ }^{5}$ T. Lopez-Rios, F. Mendoza, F.J. Garcia-Vidal, J. Sanchez-Dehesa, and B. Pannetier, Phys. Rev. Lett. 81, 665 (1988).
${ }^{6}$ F.J. Garcia-Vidal, J. Sanchez-Dehesa, A. Dechelette, E. Bustarret, T. Lopez-Rios, T. Fournier, and B. Pannetier, J. Lightwave Technol. 17, 2191 (1999).

${ }^{7}$ J.A. Porto, F.J. Garcia-Vidal, and J.B. Pendry, Phys. Rev. Lett. 83, 2845 (1999).

${ }^{8}$ U. Schröter and D. Heitmann, Phys. Rev. B 60, 4992 (1999).

${ }^{9}$ I.R. Hooper and J.R. Sambles, Phys. Rev. B 67, 235404 (2003).

${ }^{10}$ R. Dragila, B. Luther-Davies, and S. Vukovic, Phys. Rev. Lett. 55, 1117 (1985).

${ }^{11}$ H. Raether, Surface Plasmons on Smooth and Rough Gratings (Springer-Verlag, Berlin, 1988). 
${ }^{12}$ D. Sarid, Phys. Rev. Lett. 47, 1927 (1982).

${ }^{13}$ G.I. Stegeman, J.J. Burke, and D.G. Hall, Appl. Phys. Lett. 41, 906 (1982).

${ }^{14}$ R.K. Hickernell and D. Sarid, J. Opt. Soc. Am. B 3, 1059 (1986).

${ }^{15}$ T. Inagaki, M. Motosuga, E.T. Arakawa, and J.P. Goudonnet, Phys. Rev. B 32, 6238 (1985).

${ }^{16}$ S. Dupta Gupta, G.V. Varada, and G.S. Agarwal, Phys. Rev. B 36, 6331 (1987).

${ }^{17}$ J. Chandezon, M.T. Dupuis, G. Cornet, and D. Maystre, J. Opt. Soc. Am. 72, 839 (1982).
${ }^{18}$ N.P.K. Cotter, T.W. Preist, and J.R. Sambles, J. Opt. Soc. Am. A 12, 1097 (1995).

${ }^{19}$ T.W. Preist, N.P.K. Cotter, and J.R. Sambles, J. Opt. Soc. Am. A 12, 1740 (1995).

${ }^{20}$ Handbook of Optical Constants of Solids, edited by E.D. Palik (Academic, New York, 1985).

${ }^{21}$ W.L. Barnes, T.W. Preist, S.C. Kitson, and J.R. Sambles, Phys. Rev. B 54, 6227 (1996).

${ }^{22}$ W.C. Tan, T.W. Preist, and J.R. Sambles, Phys. Rev. B 62, 11134 (2000). 